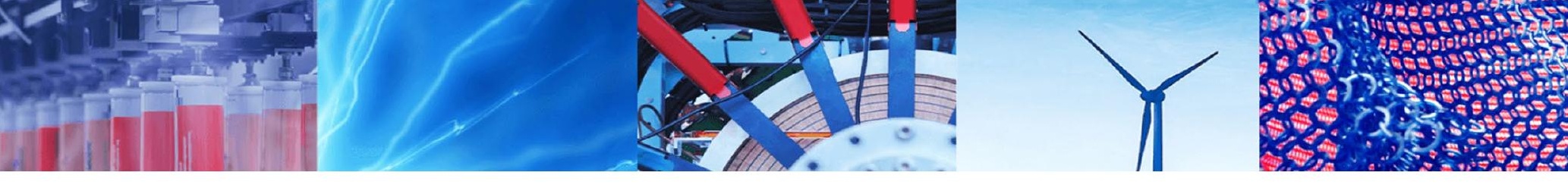

Research Article

\title{
Investigation of negative magneto-conductance properties of cobalt phthalocyanine thin films
}

\author{
S. S. Rawat ${ }^{1,2} \cdot$ A. Rana ${ }^{1,2} \cdot$ Sanjay Kumar Swami ${ }^{1} \cdot$ R. Srivastava ${ }^{1} \cdot$ C. K. Suman ${ }^{1}$ (I)
}

Received: 28 September 2019 / Accepted: 2 March 2020 / Published online: 9 March 2020

(c) Springer Nature Switzerland AG 2020

\begin{abstract}
The opto-electrical and magnetic field effects on cobalt phthalocyanine (CoPc) thin film single layer device were explored. The root mean square roughness of thin film was $5.8 \mathrm{~nm}$. The mobility was estimated for space charge limited current region in the current-voltage characteristics. Magnetic field effect on injection current was studied in terms of organic magneto-conductance. The negative magneto-conductance $(\mathrm{MC})$ was observed in CoPc thin film diode at different bias voltages. The negative values of $M C$ were found to be $10.05,8.29,6.84,4.98$ and $3.13 \%$ for applied bias voltages 1 , $2,3,4$ and $5 \mathrm{~V}$, respectively. The CoPc thin film absorbed the UV-visible wavelength range which were represented by two bands of CoPc molecule, $B$ band and $Q$ band. MC study of device under dark and light reveals the formation of trap assisted bipolarons and their quenching by the formation of photogenerated excitons. The device was checked for the resistive and capacitive behaviour by impedance spectroscopy. The contact resistance is approximately constant $\sim 66 \Omega$ at all applied bias voltages. The bulk and interface resistance vary from 1.8 to $0.6 \mathrm{k} \Omega$ and 50.06 to $1.43 \mathrm{k} \Omega$, respectively. Similarly, the bulk and interface capacitance vary from 55.81 to $10.56 \mathrm{nF}$ and 50.47 to $558.09 \mathrm{nF}$, respectively for applied bias voltages.
\end{abstract}

Keywords Exciton · Bipolaron · Magneto-conductance $\cdot$ Thin film $\cdot$ Cole-Cole plot

\section{Introduction}

Cobalt phthalocyanine (CoPc) is one of the most studied organic material due to its excellent opto-electrical properties, chemical and thermal stability [1,2]. CoPc thin film is used in organic electronics as a p-type material for organic light emitting diode, organic sensor, organic field effect transistors and organic photovoltaic [3-7]. Metal phthalocyanine compound thin film transistors are used for sensing of different volatile chemicals and gases. Phthalocyanine compound sensors confirmed fast response, high stability and good sensing behaviour [8]. Their opto-electrical properties have been discussed a lot in the scientific community but nowadays, researchers are trying to observe the magnetic field effects (MFEs) of organic semiconductor and it has opened a new window of research for organic spintronics or molecular spintronics [9-11]. It is observed that current of organic semiconductor devices can be largely tuned by a small perturbation of the magnetic field without any ferromagnetic electrodes [12]. This response of materials or devices is known as magneto-conductance (MC) or magnetoresistance (MR) $[13,14]$. The sign of organic magnetoresistance (OMAR) or organic magneto-conductance (OMC) can be negative and positive depending upon the specific nature of the organic devices $[15,16]$. The approaches of OMAR or OMC focus on charge carrier spin dynamics in the organic thin film in the MFEs [17]. In organic spintronics, organic thin film layer is inserted between two ferromagnetic materials in a vertical geometry which acts as a passive spacer. The spin

C. K. Suman, sumanck@nplindia.org| ${ }^{1}$ CSIR-National Physical Laboratory, Dr. K. S. Krishnan Marg, New Delhi 110012, India. ${ }^{2}$ Academy of Scientific and Innovative Research (AcSIR), Ghaziabad 201002, India. 
is injected and then transported in the passive spacer [18]. But the organic semiconductors show very strong MC even without ferromagnetic materials in the device [18]. Due to excited states phenomena of organic semiconductors the electroluminescent, photolumnescent and photocurrent of a device change with magnetic field [18-20]. There are several models which are used to explain the general behaviour of MC, these are: excitonic (electron-hole pair) model, bipolaron model, triplet polaron interaction model, traps and trions model etc. [21, 22]. Excitonic model can work only for bipolar devices where transport properties of both charge carriers electrons and holes are taken into consideration while bipolaron model enunciated the effect on electron and hole only devices or for bipolar device as well $[10,13,15]$. Even though, no single model can explain the origin of MC clearly so far. However, such kind of bipolaron which is two like-charges (either two electrons or two holes) located on one occupied site and are energetically unstable because of a repulsive coulomb force exerted on the pair of like charges and this bipolaron mechanism is observed in organic semiconductor [23].

In this paper, we report the investigation of MFEs on CoPc thin films with a simple device structure. The transport properties and the MC of the device are discussed with the appropriate model.

\section{Experimental details}

The single layer device of CoPc was prepared on indium tin oxide (ITO) coated glass substrate. The patterned ITO coated glass substrates were cleaned under ultrasonic bath with a solution of detergents, deionized water, acetone and isopropanol in sequence for $15 \mathrm{~min}$ and then substrates were kept in a vacuum oven at $120^{\circ} \mathrm{C}$ for $30 \mathrm{~min}$ for drying. After careful cleaning, the cleaned substrates were transferred in the $\mathrm{N}_{2}$ filled glove box and followed by UV-Ozone treatment. The active device area is $6 \mathrm{~mm}^{2}$. The CoPc thin film of $100 \mathrm{~nm}$ thickness was deposited by thermal evaporation at the rate of $\sim 0.2 \AA / s$ under the high vacuum of $\sim 10^{-6}$ mbar. Finally, a $120 \mathrm{~nm}$ thick aluminium metal electrode was thermally deposited at the rate of $\sim 1-2 \AA / s$. The device architecture of the single layer was ITO/CoPc/Al. The surface morphology of CoPc thin film was analyzed by tapping mode of atomic force microscopy (AFM) (NT-MDT Solver Pro). Optical properties of the device were measured by UV-visible spectroscopy and ellipsometry (J. A. Woolam M-2000). Current density-voltage $(J-V)$ was measured by Keithley source meter 2420 . Impedance spectroscopy measured at different DC voltages bias by Solartron, SI 1260 with the slight electrical perturbation of $100 \mathrm{mV}$ AC signal. MFE was measured by using 10-inch pole diameter DC electromagnet (Bruker) where the device was placed after fabrication. The magneto-photocurrent was generated under the light of a halogen lamp of power $\sim 10 \mathrm{~mW} / \mathrm{cm}^{2}$.

\section{Results and discussions}

The chemical structure of CoPc and device structure of the thin film are shown in the Fig. 1a. The CoPc is the neutral cobalt centered four-fold symmetry planner molecules. The orientation of CoPc thin film is influenced by the structure and roughness of the substrate that results in the flat lying, standing molecules or amorphous for the substrate. The CoPc thin film may exist in amorphous and crystalline form with polymorph $\alpha, \beta$ and $\gamma$ structures. The value of HOMO and LUMO may vary from $\sim 4.92$ to 5.20 and 2.66 to 3.40 , respectively depending upon the nature of the thin film $[1,24]$. The energy level diagram of the device shows that ITO and Al are taken as anode and cathode where the energy gap for electron is $\sim 0.80 \mathrm{eV}$ (between Aluminium and LUMO of CoPC) and for hole, the energy gap is $\sim 0.10 \mathrm{eV}$ (between ITO and HOMO of CoPc) [25-29]. There is very less probability for injection of electron in the device due to this large energy gap. Figure $1 b$ shows the schematic diagram of the device inside the electromagnet and the direction of the magnetic field from the North Pole to the South Pole of the electromagnet. The amorphous nature of the thin film was observed on the substrate as shown in the AFM image of CoPc thin film in Fig. 1c. The root mean square (RMS) roughness value of the thin film is measured to be $5.85 \mathrm{~nm}$. Although there are some spikes on the film but their density is very low and the cause for these spikes may be non-uniform deposition and/or substrate roughness. The spikes in the thin film may be due to the crystallinity of the materials as well [1]. Figure 2 shows the J-V characteristics at different temperature of the device. Clearly, there are two regions in the forward bias of the J-V characteristics. At low bias region the J-V follows ohms law $J$ a $V$ whereas, at high bias region, it follows $\mathrm{J} \mathrm{a} \mathrm{V}^{2}$. Mobility of the charge carrier is determined in the space charge limited current (SCLC) [30] region by the following expression:

$\mathrm{J}=\frac{9 \varepsilon \mu V^{2}}{8 d^{3}}$

where $\varepsilon=2.75 \times 10^{-11} \mathrm{~F} / \mathrm{m}$ [31] (experimental value), $\mu$ and $d=100 \mathrm{~nm}$ are dielectric permittivity, mobility and thickness of devices, respectively. This expression is linearly fitted in slope $\mathrm{m} \sim 2$ to analyze the mobility. It is observed that the mobility of the device is in the SCLC region at a different temperature $30,50,70,90$ and $110^{\circ} \mathrm{C}$ are found to be $4.07 \times 10^{-5}, 5.1 \times 10^{-5}, 8.1 \times 10^{-5}, 1.5 \times 10^{-4}$ 

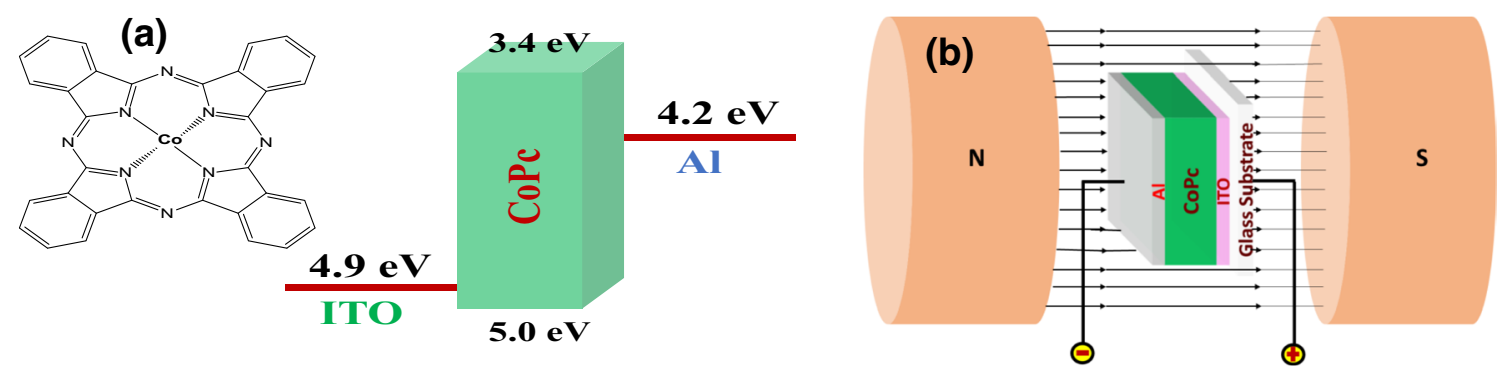

(c)

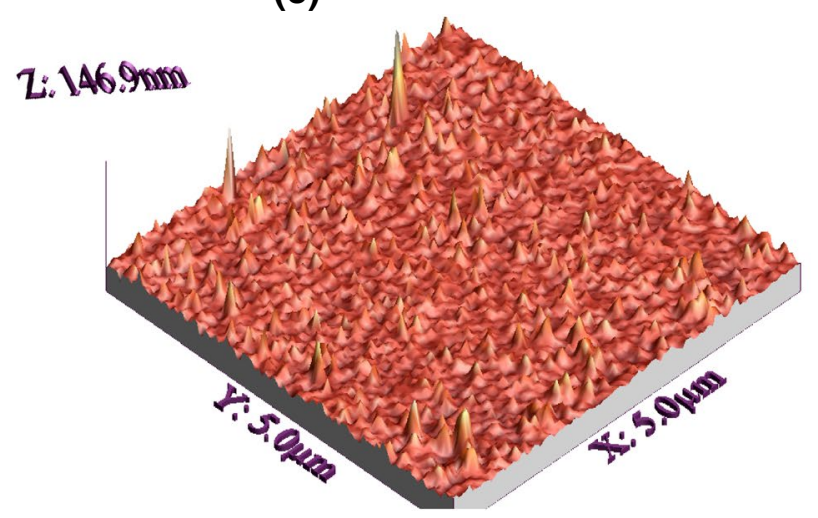

Fig. 1 a Chemical structure of CoPc and Energy level diagram of the devices, b schematic structure of device position in magnetic field, $\mathbf{c}$ AFM image of cobalt phthalocyanine thin film of $100 \mathrm{~nm}$ thickness

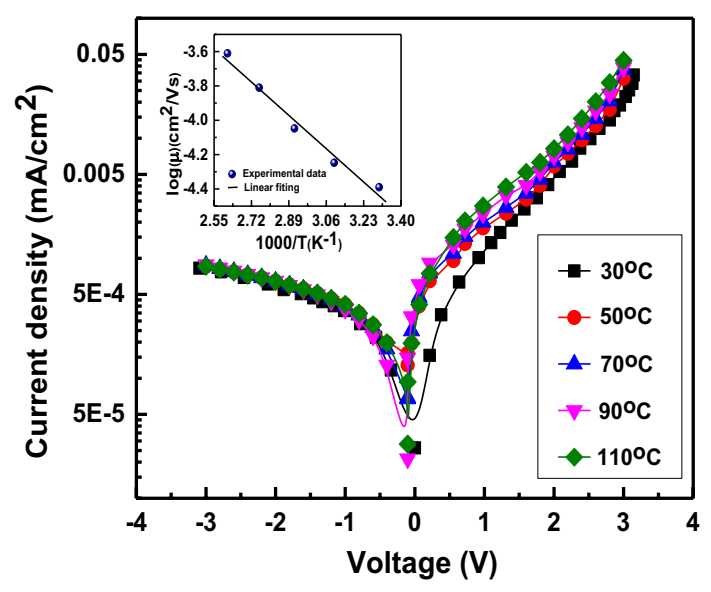

Fig. 2 Current density-voltage (J-V) characteristics of device at different temperature and (inset figure) Arrhenius plot of estimated mobility at different temperature

and $2.4 \times 10^{-4} \mathrm{~cm}^{2} / \mathrm{Vs}$, respectively. Although the current voltage characteristics does not show perfect ohmic contact but in the forward bias region the mobility may be estimated for comparison. The estimated value of mobility is very close to the earlier reported true value [32-37]. The Arrhenius plot of the calculated thermally activated mobility is shown in the inset of the Fig. 2. The Arrhenius equation for the mobility follows the equation [38]

$\mu(T)=\mu_{0} \exp \left(-E_{a} / k_{B} T\right)$

where $E_{a}$ is the activation energy and $\mu_{0}$ is the infinite temperature hole mobility, The calculated activation energy is $1.18 \mathrm{meV}$ and the infinite temperature extrapolation of the hole mobility is $\mu_{0}=6.01 \times 10^{-4} \mathrm{~cm}^{2} / \mathrm{Vs}$. Figure 3 depicts the $M C$ response of the single layer device at room temperature. The $M C$ is the relative change of current for the applied magnetic field. The MC is represented as

$M C(B)=\frac{J(B)-J(0)}{J(0)}$

where $J(B)$ and $J(0)$ terms are used for current density with and without magnetic field. The MC response is dependent on the applied electrical bias voltage of the device. The negative $\mathrm{MC}$ decreases with the increase of bias voltages. The maximum negative $M C$ was observed $\sim 10.05 \%$ at $1 \mathrm{~V}$ bias with respect to $100 \mathrm{mT}$ magnetic field. At the bias voltage of $5 \mathrm{~V}$ and the magnetic field of $100 \mathrm{mT}$, the negative $M C$ is $3.13 \%$. The negative $M C$ increases monotonically with increase of the magnetic field at all applied bias. The shape of the MC may be described by the empirical nonLorentzian function 


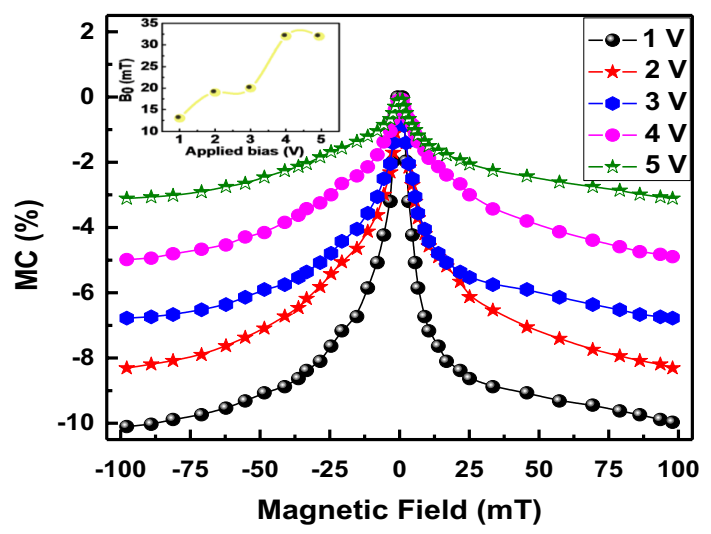

Fig. 3 Magneto-conductance (MC) versus magnetic field at different bias voltages and (inset figure) variation of FWHM $\left(B_{0}\right)$ with applied bias

$M C(B)=M C_{\infty} \frac{B^{2}}{\left(B_{0}+|B|\right)^{2}}$

where $B_{0}$ is the full width at half maxima (FWHM) and the $M C_{\infty}$ is $M C$ at infinity magnetic field. The FWHM depends on the full range of magnetic field because the $M C$ is not saturated. The inset of Fig. 3 shows that FWHM is increased with applied bias voltage. The estimated FWHM value for low applied bias is $\sim 14 \mathrm{mT}$ and increased up to $\sim 32 \mathrm{mT}$ almost double at the high bias $5 \mathrm{~V}$. The FWHM has a clear effect of bias voltages and may be correlated to the charge dynamics of the device. In general, the spin precession and electronic spin-spin interaction may be the origin of MC.
Figure 4a shows the UV-Visible optical absorption spectra of CoPc thin film in the wavelength range of $300-900 \mathrm{~nm}$. There is a B band in the ultraviolet region due to $d \rightarrow \Pi^{*}$ transitions involving the central cobalt atom and $Q$ band in the visible region due to $\Pi \rightarrow \Pi^{*}$ transitions, respectively. The Q-band is highly localized on the phthalocyanine ring, and deeply related to the surrounding of the phthalocyanine molecule of the conjugated macrocycle of $18 \pi$-electrons $[1,24]$. Maximum absorption peak in the ultraviolet and visible region are observed at $\sim 322.61$ and $\sim 610.82 \mathrm{~nm}$. Figure $4 \mathrm{~b}$ shows the refractive index and extinction coefficient behavior of thin film for the wavelength range $250-900 \mathrm{~nm}$. Refractive index varies from $\sim 1.5$ to $\sim 2.2$ and extinction coefficient varies from $\sim 0.3$ to $\sim 0.5$ in the studied wavelength range. The nature of extinction coefficient over the wavelength range from 300 to $700 \mathrm{~nm}$ is almost same as reported earlier [1]. Negative extinction coefficient is also observed in the wavelength range of 700-1000 $\mathrm{nm}$ which may be due to high reflection of optical incident wave $[39,40]$. The CoPc thin film shows broad absorption of light that may generate photocurrent. The photocurrent will affect the nature of MC. The MC of any devices or materials show the change of electrical response to an external magnetic field depending upon the spin-dependent scattering at the interface and spin-polarized carrier transport in the spacer [41]. Figure 5 a shows the semi log current-voltage characteristics of the device in light and dark. Both the dark and light current response of the device are almost same, only there is an increase of current in the mid and high value of applied bias under light. Both the curve have ohmic and $\mathrm{SCLC}$ region as already discussed in the Fig. 2. Figure $5 \mathrm{~b}-\mathrm{d}$
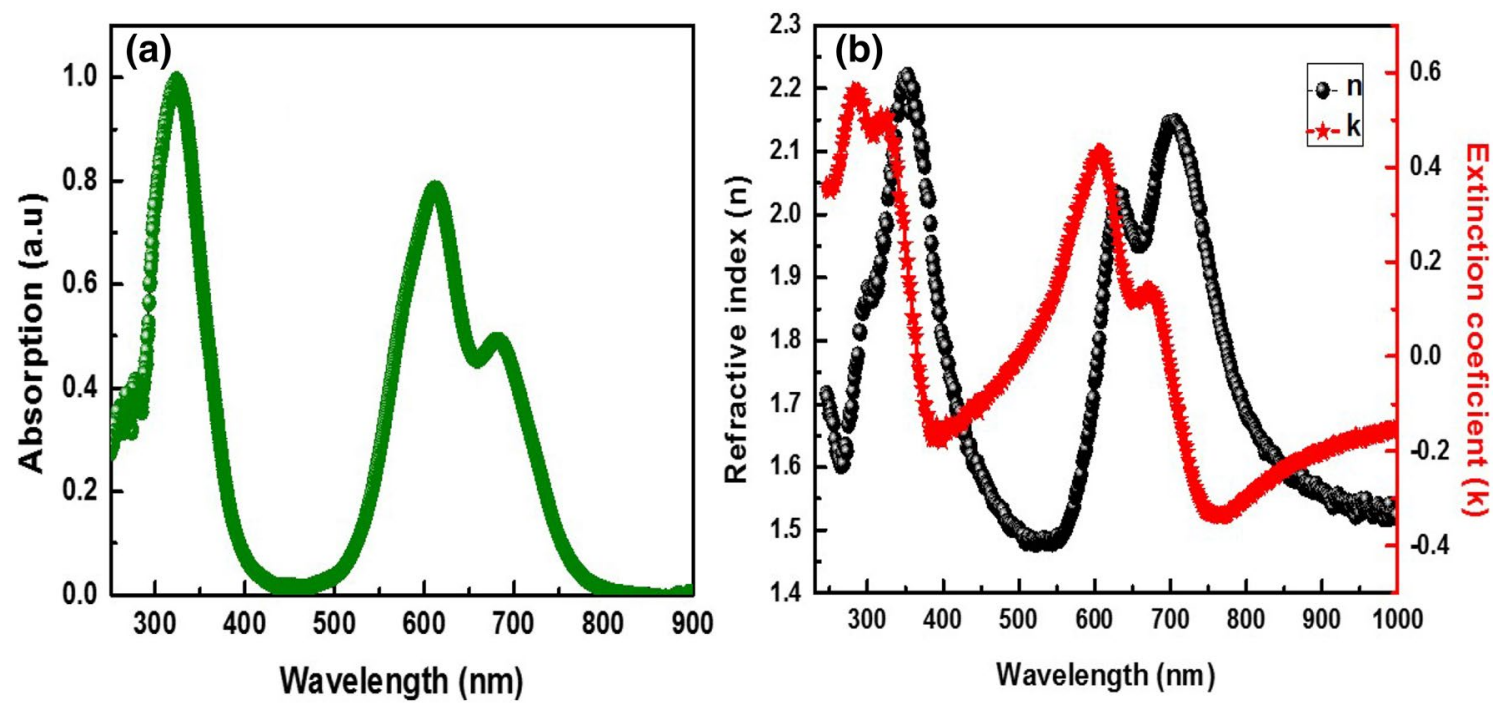

Fig. 4 a Absorption spectrum of CoPc thin film versus wavelength, b Refractive index and Extinction coefficient of CoPc thin films versus wavelength 

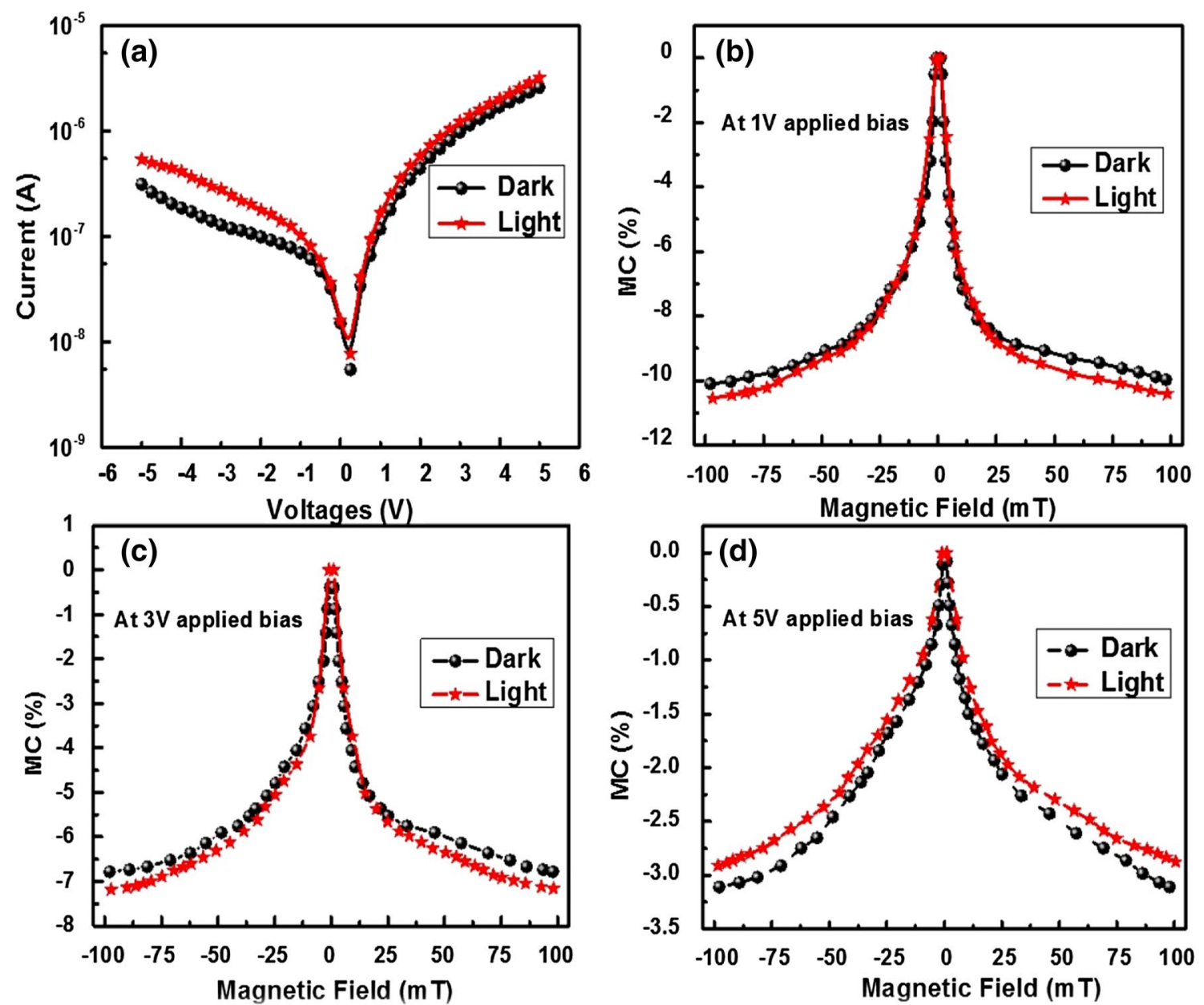

Fig. 5 a Current Voltage characteristics under light and dark b comparison of dark and under light magneto-conductance (MC) at applied bias $1 \mathrm{~V} \mathbf{c}$ at applied bias $3 \mathrm{~V} \mathbf{d}$ at applied bias $5 \mathrm{~V}$

show the MC comparison in dark and under the light of the devices at applied bias of 1,3 and $5 \mathrm{~V}$, respectively. Under light, the current of the devices is contributed from electrically injected carriers $\left(l_{i}\right)$ and photogenerated charges $\left(I_{p}\right)$

$I=I_{i}+I_{p}$

The injected current $\left(l_{i}\right)$ is due to leakage at low voltages and from regular transport near or above built in voltages. The MC under light is influenced by the space charge and traps. Since the MC either in dark or under light is negative; the physical process may be the trap assisted bipolaron formation [6]. The trap assisted bipolarons formation open the transport path and assist carrier to hop in the absence of an external magnetic field. At the same time when there is an external magnetic field, the bipolaron is spin blocked because the same field may be experienced by the spin of the positive polarons. Hence the bipolaron path may be blocked for hole hopping that results in reduced current or negative MC. The increase in bias voltage reduces the number of traps by filling them. So the number of bipolarons are increased at high bias voltages. Hence the negative $M C$ is proportional to the applied bias as already discussed in FWHM nature of Fig. 3. The possibility of bipolaron quenching at high applied bias is due to the injection of electrons from other electrodes. Under light, there are photo excited state excitons or electron-hole pairs in the devices. These excitons may affect the bipolaron formation on the trap sites. The dissociation of excitons increases the current in the devices. The comparison of MC under light and dark have been shown by Fig. 5b-d. At low voltages, the $M C$ is enlarged. At low bias voltages, the traps are being filled that promote bipolaron formation on the trap sites. At high voltages the MC decreases under light, this may be attributed to the interaction of traps with photogenerated excitons. The exciton can react with trap sites occupied by the holes. The reaction will result in charge excitons like metastable pairs that reduce the bipolaron formation at trap sites. Hence under light, the negative MC decreases. 
Figure $6 \mathrm{a}, \mathrm{b}$ show the real and imaginary form of the $A C$ impedance of CoPc thin film device. The sum of the real and imaginary part of impedance can be written in complex form as [42]

$\mathrm{Z}(\mathrm{f})=\operatorname{ReZ}(\mathrm{f})+\operatorname{ImZ}(\mathrm{f})=\left[R_{\mathrm{s}}+\frac{\mathrm{R}_{1}}{1+\omega 2 R_{1}^{2} C_{1}^{2}}\right]-\left[\frac{\omega R_{1}^{2} C_{1}}{1+\omega 2 R_{1}^{2} C_{1}^{2}}\right]$

where $R_{s}$ and $R_{1}$ represent contact and bulk resistance of the device, respectively and $C_{1}$ represents the bulk capacitance. The Eq. (6) represents the equivalent circuit parameters for one semicircle of Cole-Cole plot. Similarly, for two semicircles one more component of interface resistance and capacitance part are added to Eq. (6). The flat frequency independent region of ReZ corresponds to bulk resistance and contact resistance; in low and high frequency part of Fig. 6a. The ReZ is frequency dependent in the middle part of the frequency range $\left(\sim 10^{2}-10^{5} \mathrm{~Hz}\right)$. From the Fig. 6a, it is observed that the value of bulk resistance is decreasing when the bias voltage increases due to efficient charge transport towards the electrodes in the device [43]. Figure $6 \mathrm{~b}$ shows the peak value of $\operatorname{ImZ}$ with respect to frequency. The peak of $\operatorname{ImZ}(f)$ is proportional to bulk resistance given by the following equation:

$\operatorname{ImZ}=\mathrm{R}_{1}\left[\frac{\omega \tau}{\left(1+\omega^{2} \tau^{2}\right)}\right]$

The peak value of $I m Z$ is decreased and shifted to a higher frequency with the increase of bias voltage [44]. The frequency at which the value of $I m Z$ is maximum called the relaxation frequency and the time corresponds to relaxation time $(\tau)$ [45]. So the relaxation time of the charge

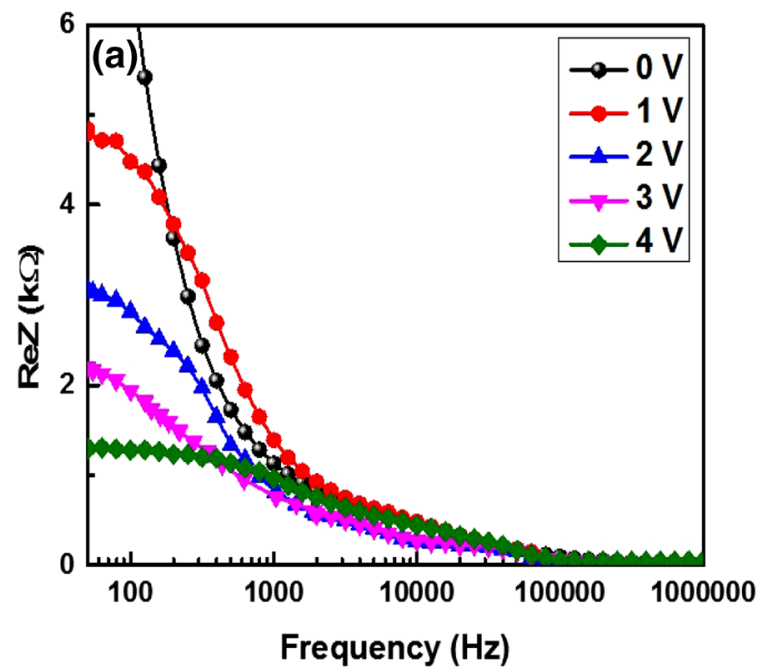

carrier is decreased with an increase of bias voltage. Figure 7 shows two semicircles in the Cole-Cole plot of the device at a different bias. The low frequency and high frequency semicircles are due to the interfacial and bulk traps [46]. It can be modelled as a two electrical parallel RC circuit in series, one for bulk resistance and another one may be interfacial resistance. The contact resistance $\left(R_{s}\right)$ is approximately constant $66 \Omega$. Bulk resistance $\left(R_{1}\right)$ and interface resistance $\left(R_{2}\right)$ vary from 1.8 to $0.6 \mathrm{k} \Omega$ and 50.06 to $1.43 \mathrm{k} \Omega$, respectively. Bulk capacitance $\left(C_{1}\right)$ and interface capacitance $\left(C_{2}\right)$ vary from 55.81 to $10.56 \mathrm{nF}$ and 50.47 to $558.09 \mathrm{nF}$, respectively with respect to under studied bias voltage. The calculated values of different resistance and capacitance at different bias voltages are listed in Table 1.

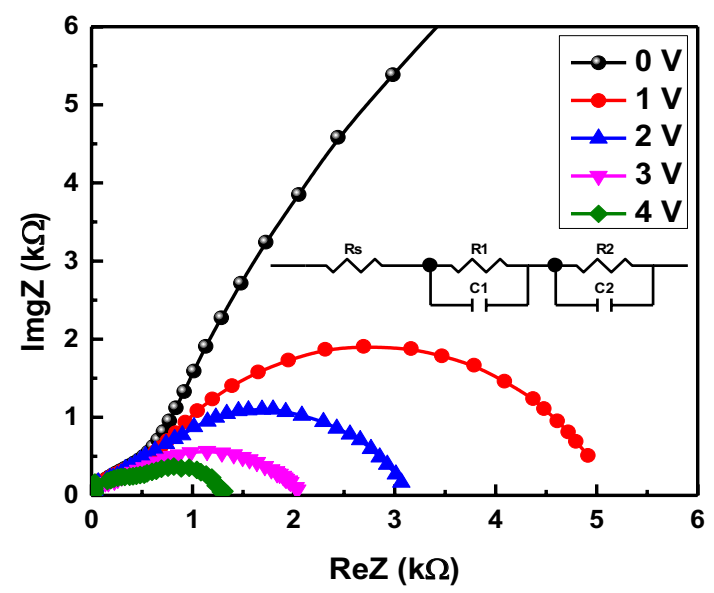

Fig. 7 Cole-Cole plot of device at different applied bias voltages

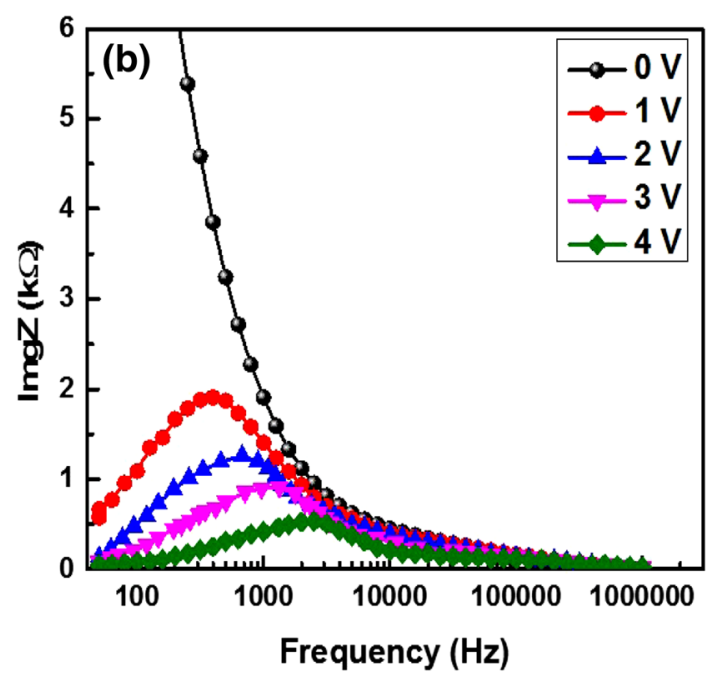

Fig. 6 Variation of impedance $\mathbf{a}$ real and $\mathbf{b}$ imaginary part of single layer device with frequency for different applied bias voltages 
Table 1 Calculated values of $R_{s^{\prime}}, R_{1}, R_{2}, C_{1}$ and $C_{2}$ of device at different bias voltages

\begin{tabular}{lllllr}
\hline DC bias $(\mathrm{V})$ & $\mathrm{R}_{\mathrm{s}}(\Omega)$ & $\mathrm{R}_{1}(\mathrm{k} \Omega)$ & $\mathrm{C}_{1}(\mathrm{nF})$ & $\mathrm{R}_{2}(\mathrm{k} \Omega)$ & $\mathrm{C}_{2}(\mathrm{nF})$ \\
\hline 0 & 66.05 & 1.8 & 55.81 & 50.06 & 50.47 \\
1 & 66.34 & 1.1 & 22.94 & 05.02 & 634.40 \\
2 & 66.76 & 0.9 & 44.41 & 03.09 & 1030.16 \\
3 & 66.89 & 0.8 & 39.71 & 02.06 & 973.01 \\
4 & 66.42 & 0.6 & 10.56 & 01.43 & 558.09 \\
\hline
\end{tabular}

\section{Conclusions}

The investigation of negative $M C$ in the single layer CoPc thin film device was discussed. The CoPc thin film has the RMS roughness of $5.85 \mathrm{~nm}$. The estimated SCLC mobility of CoPc thin film is $4.07 \times 10^{-5} \mathrm{~cm}^{2} / \mathrm{Vs}$. The estimated mobility at different temperature follows the general Arrhenius equation. The absorption spectra of thin film show B-band and Q-band in ultraviolet and visible wavelength region. The maximum value of negative $M C$ decreases with applied bias. The negative MC may be due to the formation of trap assisted bipolaron. The negative $M C$ effect and bipolaron formation have been supported by the MC under light. The exciton formation and their contribution for reducing the negative $M C$ is confirmed. The device impedance and the Cole-Cole plot show the relaxation phenomena and electrical circuit design of the device. The device is modelled by the two RC circuits in series, one for bulk and other for interface at the metal contact.

Acknowledgements The authors gratefully acknowledge the Department of Science and Technology (DST), India for providing financial support (DST-Inspire Fellowships) and Director of CSIR-National Physical Laboratory, New Delhi for providing the research facilities.

\section{Compliance with ethical standards}

Conflict of interest On behalf of all authors, the corresponding author states that there is no conflict of interest.

\section{References}

1. Nahass MME, Gohary ZE, Soliman HS (2003) Structural and optical studies of thermally evaporated CoPc thin films. Opt Laser Technol 35:523

2. Wojdyła M, Rẹbarz M, Bała W, Derkowska B, Łukasiak Z (2008) Optical properties of vacuum sublimed cobalt phthalocyanine (CoPc) thin layers. Mol Cryst Liq Cryst 485:974

3. Sizun T, Bouvet M, Chen Y, Suisse J, Barochi G, Rossignol J (2011) Differential study of substituted and unsubstituted cobalt phthalocyanines for gas sensor applications. Sensors Actuators B. Chem. 159:163
4. Chaturvedi N, Swami SK, Dutta V (2016) Electric field assisted spray deposited $\mathrm{MoO}_{3}$ thin films as a hole transport layer for organic solar cells. Sol Energy 137:379

5. Özer LM, Özer M, Altindal A, Özkaya AR, Salih B, Bekaroğlu Ö (2013) Synthesis, characterization, OFET and electrochemical properties of novel dimeric metallophthalocyanines. Dalt Trans 42:6633

6. Kao PC, Chu SY, You ZX, Liou SJ, Chuang CA (2006) Improved efficiency of organic light-emitting diodes using CoPc buffer layer. Thin Solid Films 498:249

7. Kwong CY, Djurisic AB, Lam LSM, Chan WK (2003) Phthalocyanine based Schottky solar cells. Org. Photovoltaics III 4801:7

8. Polat MP, Akyüz D, Yasemin Yenilmez H, Koca A, Altindal A, Bayr ZA (2019) Sensing alcohol vapours with novel unsymmetrically substituted metallophthalocyanines. Dalt Trans 48:9194

9. Atxabal A, Ribeiro M, Parui S, Urreta L, Sagasta E, Sun X, Llopis R, Casanova F, Hueso LE (2016) Spin doping using transition metal phthalocyanine molecules. Nat Commun 7:1

10. Chen L, Lei Y, Zhang Q, Xiong Z (2015) Negative magnetoconductance effects in amorphous copper phthalocyanine thin film: trap-assisted bipolaron formation. J Mater Chem C 3:12056

11. Cabero-Zabalaga MA, Wei J, Yang H, Fan BB, Sun Y, Zhao W (2017) Unraveling the characteristic shape for magnetic field effects in polymer-fullerene solar cells. ACS Omega 2:7777

12. Sheng Y, Nguyen TD, Veeraraghavan G, Mermer Ö, Wohlgenannt $M$ (2007) Effect of spin-orbit coupling on magnetoresistance in organic semiconductors. Phys Rev B 75:045213

13. Bobbert PA, Nguyen TD, Van Oost FWA, Koopmans B, Wohlgenannt M (2007) Bipolaron mechanism for organic magnetoresistance. Phys Rev Lett 99:216801

14. Hu B, Yan L, Shao M (2009) Magnetic-field effects in organic semiconducting materials and devices. Adv Mater 21:1500

15. Bloom FL, Wagemans W, Kemerink M, Koopmans B (2007) Separating positive and negative magnetoresistance in organic semiconductor devices. Phys Rev Lett 99:257201

16. Hu B, Wu Y (2007) Tuning magnetoresistance between positive and negative values in organic semiconductors. Nat Mater 6:985

17. Oviedo-Casado S, Urbina A, Prior J (2017) Magnetic field enhancement of organic photovoltaic cells performance. Sci Rep 7:4297

18. Huang WS, Xu ZR, Hu B, Guo TF, Huang JCA, Wen TC (2013) Magnetoconductance responses of triplet polaron pair charge reaction in hyperfine coupling regime. Appl Phys Lett 103:1

19. Nguyen TD, Rybicki J, Sheng Y, Wohlgenannt M (2008) Device spectroscopy of magnetic field effects in a polyfluorene organic light-emitting diode. Phys Rev B 77:035210

20. Gautam BR, Nguyen TD, Ehrenfreund E, Vardeny ZV (2013) Magnetic field effect spectroscopy of $\mathrm{C}_{60}$-based films and devices. J Appl Phys 113:143102

21. Gobbi M, Orgiu E (2017) The rise of organic magnetoresistance: materials and challenges. J Mater Chem C 5:5572

22. Cox M, Janssen P, Zhu F, Koopmans B (2013) Traps and trions as origin of magnetoresistance in organic semiconductors. Phys Rev B 88:035202

23. Behrends J, Schnegg A, Lips K, Thomsen EA, Pandey AK, Samuel IDW, Keeble DJ (2010) Bipolaron formation in organic solar cells observed by pulsed electrically detected magnetic resonance. Phys Rev Lett 105:12

24. Joseph B, Menon CS (2008) Studies on the optical properties and surface morphology of cobalt phthalocyanine thin films. $E$ J Chem 5:86

25. Ma Z, Zhao J, Wang X, Yu J (2016) Effect of bulk and planar heterojunctions based charge generation layers on the performance of tandem organic light-emitting diodes. Org Electron 30:136

26. Radbeh R, Parbaile E, Bouclé J, Di Bin C, Moliton A, Coudert V, Rossignol F, Ratier B (2010) Nanoscale control of the network 
morphology of high efficiency polymer fullerene solar cells by the use of high material concentration in the liquid phase. Nanotechnology 21:035201

27. Pode $R$ (2011) On the problem of open circuit voltage in metal phthalocyanine/C60 organic solar cells. Adv. Mater. Lett. 2:3

28. Terao Y, Sasabe H, Adachi C (2007) Correlation of hole mobility, exciton diffusion length, and solar cell characteristics in phthalocyanine/fullerene organic solar cells. Appl Phys Lett 90:103515

29. Petraki F, Peisert H, Biswas I, Chassé T (2010) Electronic structure of Co-phthalocyanine on gold investigated by photoexcited electron spectroscopies: indication of Co ion-metal interaction. J Phys Chem C 114:17638

30. Wang ZB, Helander MG, Greiner MT, Qiu J, Lu ZH (2010) Controlling carrier accumulation and exciton formation in organic light emitting diodes. J Appl Phys 107:3

31. Samanta S, Aswal DK, Singh A, Debnath AK, Senthil Kumar M, Hayakawa Y, Gupta SK, Yakhmi JV (2010) Bias and temperature dependent charge transport in high mobility cobalt phthalocyanine thin films. Appl Phys Lett 96:2008

32. Malik TGA, Latif RMA (1996) Transport properties in oxygendoped cobalt phthalocyanine thin film devices. Thin Solid Films 286:277

33. Mahapatro AK, Ghosh S (2002) Schottky energy barrier and charge injection in metal/copper-phthalocyanine/metal structures. Appl Phys Lett 80:4840

34. Röhr JA, Moia D, Haque SA, Kirchartz T, Nelson J (2018) Exploring the validity and limitations of the Mott-Gurney law for chargecarrier mobility determination of semiconducting thin-films. J Phys: Condens Matter 30:105901

35. Wang W, Ji Z, Lu C, Wang L, Xu G, Li L, Liu M (2014) An organic rectifier diode based on poly-pyrrole (PPY) electrode. Proceedings of the-2014 IEEE 12th international conference on solidstate and integrated-circuit technology ICSICT 2014

36. Sussman A (1967) Electrical properties of copper phthalocyanine thin films as influenced by the ambient. J Appl Phys 38:2748

37. Gravano S, Hassan AK, Gould RD (1991) Effects of annealing on the trap distribution of cobalt phthalocyanine thin films. Int J Electron 70:477
38. Zufle S, Altazin S, Hofmann A, Jager L, Neukom MT, Brutting W, Ruhstaller B (2017) Determination of charge transport activation energy and injection barrier in organic semiconductor devices. J Appl Phys 122:115502

39. Djurišić AB, Kwong CY, Lau TW, Liu ZT, Kwok HS, Lam LSM, Chan WK (2003) Spectroscopic ellipsometry of metal phthalocyanine thin films. Appl Opt 42:6382

40. Chen YJ, Lee CC, Chen SH, Flory F (2013) Extra high reflection coating with negative extinction coefficient. Opt Lett 38:3377

41. Klemm P, Bange S, Malissa H, Boehme C, Lupton JM (2018) Temperature and current dependence of the magnetoresistive behavior of poly(styrene-sulfonate)-doped poly(3,4-ethylenedioxythiophene) (PEDOT:PSS). J Photon Energy 8:032216

42. Ramar M, Rawat SS, Srivastava R, Dhawan SK, Suman CK (2016) Impact of Cross Linking Chain of N, N-bis(napthalen-|-y|)-N, Nbis (phenyl)-benzidine on Temperature dependent Transport Properties. Adv Mater Lett 7:783

43. Zhang Y, Li L, Yuan S, Li G, Zhang W (2013) Electrical properties of the interfaces in bulk heterojunction organic solar cells investigated by electrochemical impedance spectroscopy. Electrochim Acta 109:221

44. Padmamalini $\mathrm{N}$, Ambujam $\mathrm{K}$ (2016) Impedance and modulus spectroscopy of $\mathrm{ZrO}_{2}-\mathrm{TiO}_{2}-\mathrm{V}_{2} \mathrm{O}_{5}$ nanocomposite. Karbala Int J Mod Sci 2:271

45. Arredondo B, Romero B, Del Pozo G, Sessler M, Veit C, Würfel $U$ (2014) Impedance spectroscopy analysis of small molecule solution processed organic solar cell. Sol Energy Mater Sol Cells 128:351

46. Miller KA, Yang RD, Hale MJ, Park J, Fruhberger B, Colesniuc CN, Schuller IK, Kummel AC, Trogler WC (2006) Electrode independent chemoresistive response for cobalt phthalocyanine in the space charge limited conductivity regime. J Phys Chem B 110:361

Publisher's Note Springer Nature remains neutral with regard to jurisdictional claims in published maps and institutional affiliations. 